EPJ Web of Conferences 59, 10001 (2013)

DOI: $10.1051 /$ epjconf/20135910001

(C) Owned by the authors, published by EDP Sciences, 2013

\title{
Gas X-pinch: The progress, technology and results
}

\author{
V.I. Zaitsev ${ }^{1}$, I.A. Barykov ${ }^{1}$, A.S. Boldarev² and O.G. Olkhovskaya ${ }^{2}$ \\ ${ }^{1}$ State Research Center of Russian Federation Troitsk Institute for Innovation and Fusion \\ Research, Moscow reg., Troitsk, Russia \\ ${ }^{2}$ Keldysh Institute of Applied Mathematics, Russian Academy of Sciences, Moscow, Russia
}

\begin{abstract}
The plasma pinch on the basis of a gas jet with special density distribution is described. The gas jet was produced by means of a pulse gas valve with a nozzle that was capable of forming a gas flow with a maximum density in the center of the discharge gap. The diagnostic techniques involved measurements of the discharge current and the pinch voltage, the size of the radiation area and the X-ray intensity. The experiments with different gases $\left(\mathrm{Ar}, \mathrm{Ne}\right.$ and $\mathrm{N}_{2}$ ) showed that as a result of the discharge at this gas distribution the hot plasma area of "point" form (its typical size is $100 \mu \mathrm{m}$ and lifetime 3-5 ns) arises in the middle of the discharge gap. This area is the X-ray source of energy of quanta $50-5000 \mathrm{eV}$. The radiation spectrum depends on the gas type. The experimental results are presented.
\end{abstract}

\section{INTRODUCTION}

Along with some investigations into the field of thermonuclear fusion in recent years high-current Z-pinches have found increasing application as the X-radiation sources. Depending on the task, various methods of pinch formation are applied which differ in both electric parameters (value and rise time of pinch current) and load characteristics (material and initial mass distribution). To create a point-like $\mathrm{X}$-radiation source the loads are applied that are formed by cross-shaped thin wires (X-pinch) or a profiled light material (for instance, agar-agar) [1, 2]. As a result of a high-current discharge $\left(>10^{4} \mathrm{~A}\right)$ in the place of the maximum current density there appears a plasma area of micron sizes producing high-level emission of X-ray quanta. The main barrier to the application of such methods of creating "point" sources of X-ray is the need to recover the load after each shot of the facility that entails opening the vacuum chamber, next out-pumping, etc. To create an X-ray source which can operate in repetitive mode, we tried to use a gas jet instead of a solid-state X-pinch load [3].

\section{EXPERIMENTAL SETUP}

Using the specially designed ring-shaped nozzle (Fig. 1, left) in one of the electrodes, it is possible to obtain an "X-pinch-like" density distribution of the gas jet in the discharge gap, Fig. 2. This density distribution is characterised by the density maximum near the middle of the discharge gap.

The experiments were carried out on a facility with its parameters: a maximum current of $250 \mathrm{kA}$, a rise time of $1 \mu \mathrm{s}$ and a discharge gap of $1.5 \mathrm{~cm}$. The discharge gap current was generated by the discharge of an $8-\mu \mathrm{F}$ capacitor bank with an initial voltage of $20 \mathrm{kV}$ through a triggered vacuum gap. A shot is preceded by the filling of the discharge gap by a gaseous-neon jet generated by an electromagnetic valve with a nozzle Fig. 1, left.

This is an Open Access article distributed under the terms of the Creative Commons Attribution License 2.0, which permits unrestricted use, distribution, and reproduction in any medium, provided the original work is properly cited. 

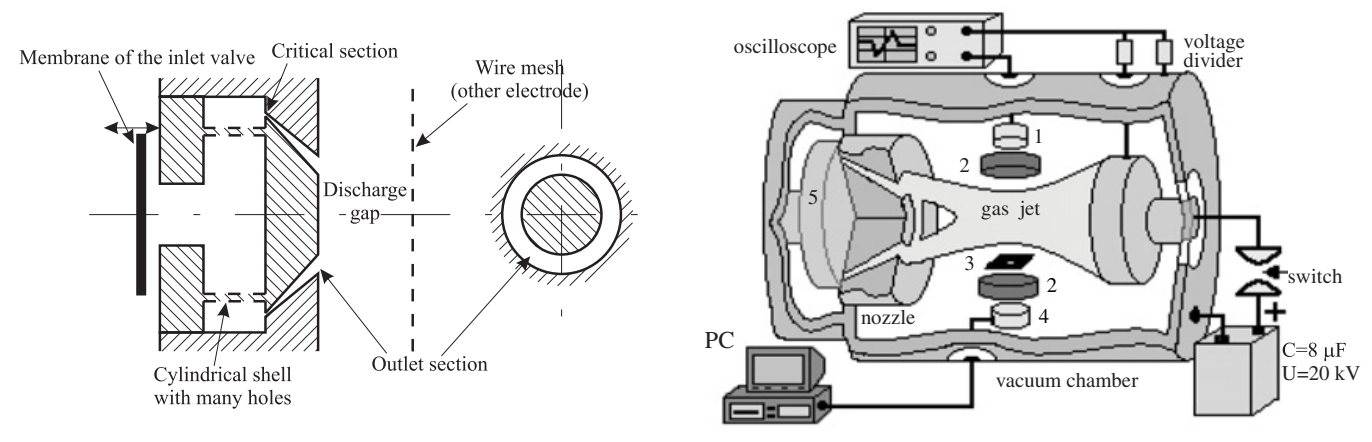

Figure 1. Left: A ring-shaped nozzle and the discharge gap. Right: Experimental setup: $1-\mathrm{X}$-ray $p$ - $i-n$ detector; 2 - filter; 3 - pinhole $(50 \mu \mathrm{m}) ; 4$ - X-ray CCD camera; 5 - gas valve.
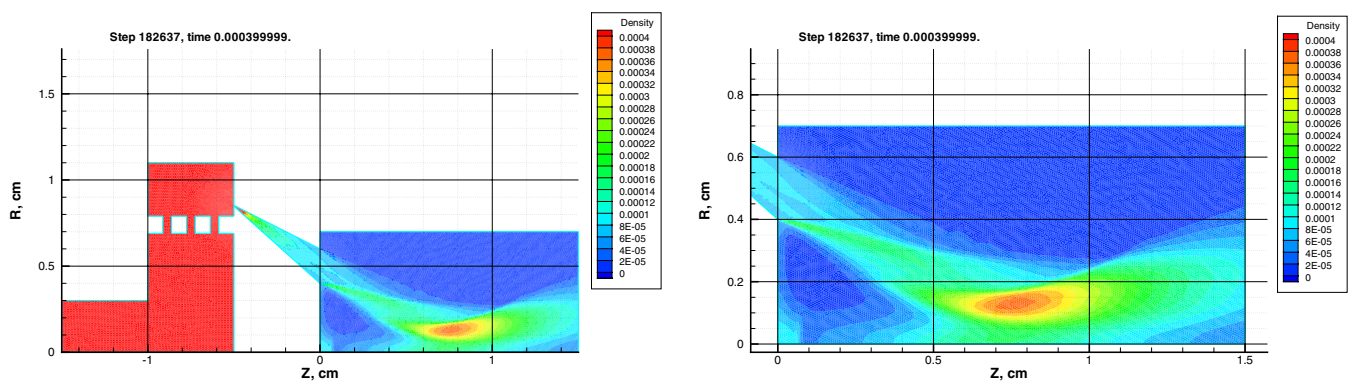

Figure 2. Density distribution for argon at initial pressure $P_{0}=5$ bar.

Since the valve had a certain dynamics of opening, the absolute gas density at the discharge gap could be regulated both by the pressure at the valve inlet and the value of time delay of opening the valve.

The experimental scheme is shown in Fig. 1, right. The diagnostic techniques involved measurements of the discharge current and the pinch voltage, the size and intensity of the X-ray radiation area. The X-ray radiation was detected by $\mathrm{Si} p$ - $i$ - $n$ diode with a time resolution of about $1.5 \mathrm{~ns}$. The sizes of X-ray source were detected by means of the pinhole picture on the CCD matrix $(1392 \times 1032$ pixels $)$. All images were taken using a filter consisting of $2.4-\mu \mathrm{m}$ Dacron $+0.85-\mu \mathrm{m}$ Al. The real-scale CCD signal was displayed on the computer monitor.

\section{EXPERIMENTAL RESULTS}

The investigations were mainly aimed at finding parameters of the pinch radiation dependent on gas density distribution along the discharge gap. At rather insignificant delays in the installation start relative to the electromagnetic valve run $(\tau=400-430 \mu \mathrm{s})$ the radiation is a train of short pulses with a great spread in time among them. On the pinhole image a series of point emitters is observed which are extended at a distance of the order of $3 \mathrm{~mm}$ along the pinch axis. At time delays of 440-460 $\mu$ s regimes are effected when a single pulse of X-radiation is observed. In Fig. 3 is a radiation pulse synchronized with the voltage on the pinch. It is possible to see that X-ray is appeared through 0.7 current rise time (approximately), and there is possibility of some adjusting of the compression regimes.

The pulse duration is of the order of $5 \mathrm{~ns}$ (Fig. 3). The total X-ray yields were $\sim 30 \mathrm{~mJ}$ for Ne and $10 \mathrm{~mJ}$ for Ar. The detector was mounted at a distance of $65 \mathrm{~cm}$ behind an aluminum filter with a thickness of $6 \mu \mathrm{m}$. The X-ray radiation was detected in the photon energy range $0.8-1.5 \mathrm{keV}$ corresponding to the emission by hydrogen- or helium-like neon ions. 

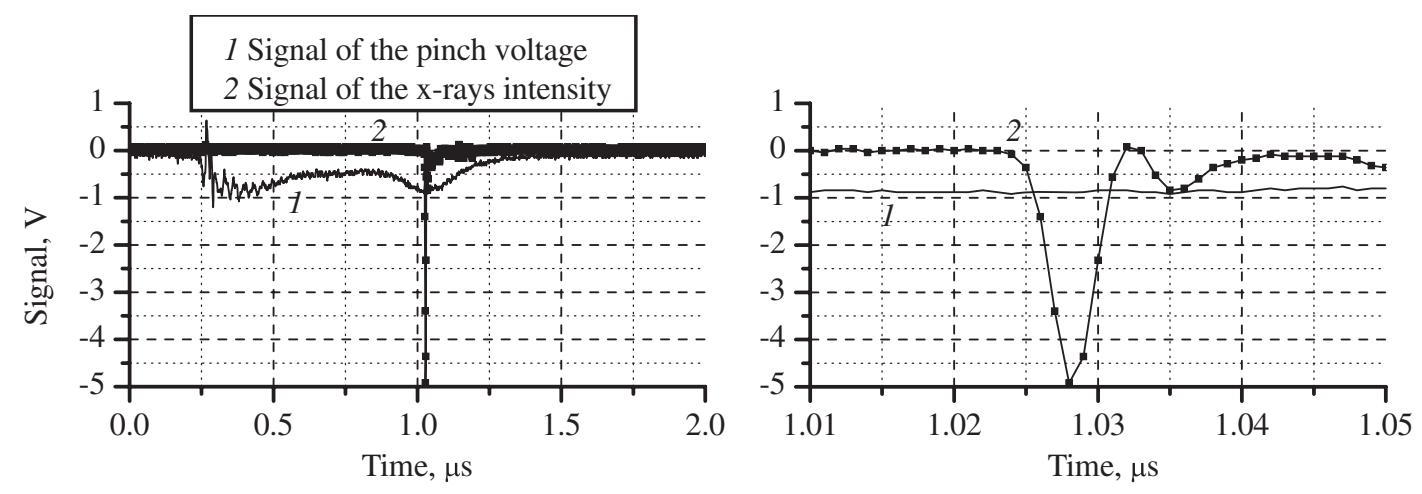

Figure 3. Measured intensity of X-rays emitted from the gas pinch.

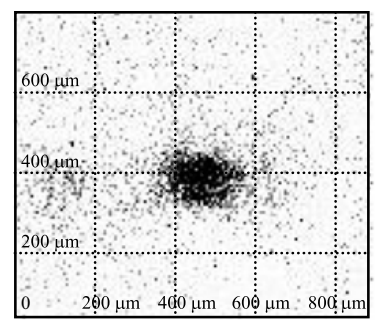

Figure 4. The pinhole picture of the gas pinch.

The one area of X-ray radiation is appeared near center of discharge gap by single X-ray pulse. On the Fig. 4 the pinhole picture of this area is shown. The real sizes of radiation area are determined from CCD picture and pinhole geometry. From shot to shot pinhole picture was in limits 200-300 $\mu \mathrm{m}$. Usually the size location along discharge axis is somewhat large. Since the object resolution was $75 \mu \mathrm{m}$, the minimum source size obtained in our experiments is not larger than $100 \mu \mathrm{m}$.

\section{MATHEMATICAL MODELLING}

To model the operation of such an experimental installation, one should model two different processes: the production of initial density configuration and its electrical implosion. In the first task, only the gas-dynamics processes are important. We solve the 2D axisymmetric Euler equations with use of finite volume TVD approximation at unstructured meshes. The use of unstructured meshes helps us to discretize the computational domains with comparatively complex geometry, for instance, when the computational domain includes the inner cavity of the nozzle and some open space near the outlet section.

The purpose of the gasdynamical modelling was to fit the shape of the nozzle to provide an "X-pinch-like" jet density distribution in the discharge gap, with the density maximum near its middle. The left part of the Fig. 1 represents the scheme of the nozzle obtained as a result. The chosen geometrical sizes of the nozzle are the result of the compromise between the desire to obtain very high Mach number (which ensures less lateral expansion of the jet and, as a result, more "sharp" density configuration) and the technological limitations which do not allow to fabricate a nozzle with too narrow critical section.

A nozzle of the type Fig. 1 which satisfies the technological limitations provides the Mach number about 6 near the nozzle outlet for gases with $\gamma=5 / 3$. At Fig. 2 the computed density profile for this 


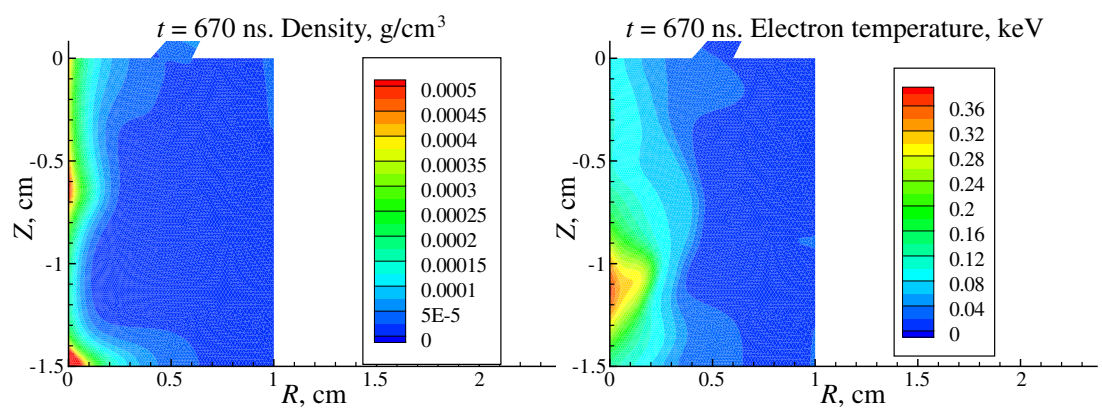

Figure 5. Results of MHD modelling of density configuration Fig. 2.

nozzle is presented. The modelling have been performed for argon at initial pressure 5 bar, the density levels are in $\mathrm{g} / \mathrm{cm}^{3}$. One can see that there is some density maximum near the middle of the discharge gap. But, in spite of extreme simplicity of the initial jet structure, the whole flow seems to be rather complicated. It has some important features that should be noted.

First of all, the density structure is not completely stationary but is subjected to some oscillations, which, however, are comparatively small and do not influence the density pattern as a whole. Then, the density maximum is not situated at the axis but at some distance from it. This is the case for various variants, with different sizes of outlet orifice, gas properties, Mach numbers etc. Upstream the density maximum, there is a zone with reverse flow along the axis. This reverse flow interacts with the solid wall (electrode) with the normal shock wave, and spreads along the electrode from the axis. Near the nozzle's outlet the interaction of two flows takes place: the backward flow spreading along the electrode hits the main flow issuing from the nozzle, this collision results in the slanted shock wave, and the main flow changes its direction.

For the modelling of electrical implosion of different loads, we use the two-temperature magnetohydrodynamic model with anisotropic dissipative processes and radiational heat transfer. At Fig. 5 the results of MHD modelling of implosion of the density configuration presented at Fig. 2 are presented. The working gas was taken to be neon (with corresponding scaling of initial density), $I=$ $A \sin (\omega t)$, where $A=0.25 \mathrm{MA}, \omega=1.57 \mu \mathrm{s}^{-1}$. Figure 5 represents the time instant which corresponds to the maximum of X-ray emission. One can see that there is a localized zone with high electron temperature, but this zone is not in the middle of the discharge gap, where the initial density profile has the maximum.

\section{CONCLUSIONS}

Optimization of the gas nozzle shape makes it possible to obtain a gas distribution with a maximum density and minimum cross section size near the discharge gap center. By gas density profiling in the discharge gap it was possible to create a point-like source of radiation in the range of energy quanta in XUV and soft X-ray area. The minimum size of the radiating area is about $100 \mu \mathrm{m}$ that, in many applications, significantly simplifies the optical scheme. The pulse radiation duration is $3-5 \mathrm{~ns}$ that allows to use the given source for detection of high-speed processes. Numerical modelling of various processes concerning the gas $\mathrm{X}$-pinch configuration give the adequate results.

\section{References}

[1] T. A. Shelkovenko, D. B. Sinars, S. A. Pikuz, D. A. Hammer, Phys. Plasmas, 8, 1305 (2001)

[2] V. V. Vikhrev, V. D. Korolev, Plasma Physics Letter, 33, 356 (2007)

[3] V. I. Zaitsev, G. S. Volkov, I. A. Barykov et al, JETP Letters, 88, 582 (2008) 